\title{
Diffusion Tensor-Based Fiber Tracking in Cervical Spinal Cord with a 3T MRI
}

\author{
M. C. $\mathrm{Ng}^{1,2}$, Y. $\mathrm{Hu}^{1}$, E. X. Wu ${ }^{3}$, E. S. Yang ${ }^{2}$, K.D. K. Luk ${ }^{1}$ \\ Department of Orthopaedics and Traumatology, \\ Jockey Chub Magnetic Resonance Imaging Centre ${ }^{2}$, \\ Department of Electrical and Electronic Engineering ${ }^{3}$, \\ The University of Hong Kong, Pokfulam, HK \\ mcng@eee.hku.hk,yhud@hkusua.hku.hk,ewu@eee.hku.hk,esyang@eee.hku.hk, \\ hrmoldk@hkucc.hku.hk
}

\begin{abstract}
The protocol for spinal DTI and tractography with high SNR and spatial resolution has been developed and tested on 6 healthy subjects and 1 CSM patient in a $3 T$ system. Fiber bundles were traced and were found running parallel to the cervical spinal cord correlating with the neuronal anatomy in normal subjects. The FA maps showed consistent low FA region connecting all spinal levels, which corresponded to the grey matter structure in anatomical imaging. Spinal DTI in CSM showed diffusivity increase at compression sites. The proposed diffusion eigenvector-based method was able to differentiate between $\lambda a$ and $\lambda r$ even when there was a drastic diffusivity change at compressed regions in CSM, which may facilitate better understanding of the pathophysiology of CSM. Our results indicated that using the current imaging and post-processing protocols, spinal DTI can be achieved with better grey/white matter contrast, high inter-subject reproducibility and diagnostic ability.
\end{abstract}

\section{Introduction}

Since the introduction of $3 \mathrm{~T}$ scanners into clinical practice, advantages of high field MRI have been extensively explored in many radiological applications. The general advantage of $3 \mathrm{~T}$ over $1.5 \mathrm{~T}$ is a doubling of the available signal-to-noise ratio (SNR), which can be traded for increased spatial resolution or shorter acquisition time. In this study, we would like to perform in-vivo diffusion tensor imaging (DTI) with tractography of the human cervical spinal cord in axial plane at a $3 \mathrm{~T}$ scanner. Tractography is a MRI technique for testing the fiber connectivity and for visualization of the orientation and structure of the fiber tracts. Spinal DTI and tractography have great potential in medical diagnosis of different spinal cord diseases like cervical spondylotic myelopathy (CSM).

CSM is a neurodegenerative disease in which the pathophysiology is still not clearly understood. Since the discovery of DTI, the diffusion change in the compression areas of CSM patients have been studied [1-4]. It was reported that there was fractional anisotropy (FA) decrease [1-3] and apparent diffusion coefficient (ADC) increase [1-4] in the compression area. Researchers suggested that the diffusion pattern change might be related to the increased permeability of the membranes due to chronic hypoperfusion and a subsequent partial destruction of the membranes [4], abnormal motion of the CSF [4] or histopathologic changes, including gliosis, microcystic degeneration, and extracellular edema [3]. However, from some studies, it was also found that a FA increase was sometimes detected at the level of compression $[4,5]$, which seems to be different from other results $[2,3]$. On the other hand, most of the spinal DTI studies were carried out in sagittal plane which suffered from partial volume effect by the surrounding cerebrospinal fluid (CSF) as stated by the authors $[3,4]$. In case of CSM patients whose spinal cord are compressed by the bulging/herniated disc or osteophytes, the partial volume effect is even greater which adversely affects the analysis and may lead to low specificity for lesion detection [2].

In conventional DT calculation, after the generation of the diffusion tensor matrix from a series of diffusion weighted images, the three eigenvalues or diffusivities $\left(\lambda_{1}, \lambda_{2}\right.$ and $\left.\lambda_{3}\right)$ are calculated by matrix diagonalization. The axial diffusivity ( $\lambda \mathrm{a}$ ) is always defined as the maximum eigenvalue $\left(\lambda_{1}\right)$, and the radial diffusivity ( $\lambda r$ ) as the mean of $\lambda_{2}$ and $\lambda_{3}\left(\lambda_{(2+3) / 2}\right)$. However, in case of CSM, the serious compression always leads to a 
change in the principal diffusion vector directions [4] and the diffusivity change is so great that it is not straightforward to differentiate between $\lambda \mathrm{a}$ and $\lambda \mathrm{r}$ by using conventional DT definition. In this study, we would like to propose a diffusion eigenvector-based method to differentiate between $\lambda \mathrm{a}$ and $\lambda \mathrm{r}$ in CSM

The incentive of this study is to establish an axial DTI protocol with spinal tractography with small motion artifact, high SNR and spatial resolution, which can be used to establish a DTI model for diagnosis of spinal cord diseases like CSM. However at high field, the susceptibility problem becomes more severed and overcoming the susceptibility artifacts from the tissue boundary is challenging. In this study, we used small matrix size and reduced FOV to attain a short TE protocol to circumvent the problem. The data were then compared with the anatomical imaging to show the clear grey/white contrast obtained in DTI. The protocol was also applied to a CSM patient to show the abnormal diffusion changes in the compression area. In order to understand more about the pathophysiology of CSM, different DT parameters at different spinal levels were studied. Diffusion eigenvector-based method was used to differentiate between $\lambda \mathrm{a}$ and $\lambda \mathrm{r}$ for better understanding of the mechanism of CSM.

\section{Method}

DTI studies were carried out on 6 healthy volunteers and 1 CSM patient with a 3T MRI scanner (Philips Achieva) with cardiac triggering. Three DT scanning was performed on the CSM patient for reproducibility test. CTL spine coil was used to enclose the cervical region. The pulse sequence used was spin-echo echo-planar imaging (SE-EPI) with 15 diffusion gradient directions (diffusion weighing $=0$ \& $400 \mathrm{~mm}^{-2} \mathrm{~s}$ ). The pulse sequence parameters were $\mathrm{TR}=5$ heart beats, $T E=55$, voxel size $=1 \times 1.26 \times 5 \mathrm{~mm}^{3}$, slice spacing $=1 \mathrm{~mm}, \quad \mathrm{NEX}=3$ and phase encoding direction $=$ anterior/posterior. Spectral Presaturation with Inversion Recovery (SPIR), spatial saturation, high-order shimming and and SENSE Constant intensity Level AppeaRance (CLEAR) were employed. The DT scanning took less than 5 minutes. Within the scanning period, the subjects were instructed not to swallow to minimize the motion artifact. Tractography was performed on-site using the diffusion tensor tractography software, PRIDE V4 [6]. Seed points were placed inside the spinal cord on the middle slice for fiber tracking. Streamlines algorithm was used in tractography with anisotropy and direction threshold equal to 200 and 850 respectively. Anatomical axial T2-weighted (T2W) imaging was also conducted for the comparison with the DT images. The imaging stack position was the same as the one in DTI.

On the other hand, Quantitative study was performed in the CSM patient to study the diffusion pattern changes. Tensor calculation was achieved by DTIStudio [7] and ROIs were drawn manually in each slice to cover the gray and white matter of the spinal cord. Boundary voxels were excluded to minimize the partial volume effect. The DT parameters, FA, tensor trace (tt), $\lambda_{1}$ and $\lambda_{(2+3) / 2}$ were examined at different spinal levels.

In order to differentiate between $\lambda \mathrm{a}$ and $\lambda \mathrm{r}$ in CSM, diffusion eigenvector-based method was adopted. It was assumed that all the axial diffusion components ran along the spinal cord ( $Z$ direction) and the radial components lied in the $X-Y$ plane. By sorting the $Z$ component of the diffusion eigenvectors, the corresponding eigenvalue with the diffusion eigenvector having the greatest absolute $Z$ component was regarded as $\lambda \mathrm{a}$. The mean of the other two eigenvalues was regarded as $\lambda \mathrm{r}$. $\lambda \mathrm{a}$ and $\lambda \mathrm{r}$ within the spinal cord at different levels calculated by our diffusion eigenvector-based method were compared with the conventional method.

\section{Results}

Spinal DTI and tractography were performed in 3T system. Figures 1a,b,e,f show the 3D colour-coded FA maps of 4 non-contiguous slices in one of the healthy subjects. The blue colour in the cord means that the fiber runs in a superior/inferior direction. Low FA regions at the anterior part of the spinal cord were detected consistently across subjects. Figures 1c,d,g,h show the T2W images at the same positions as the DTI and figure 2 shows the fiber bundles traced in the healthy cervical spinal cord.

In case of CSM, Figure 3a shows the T2-weighted sagittal image overlaid by the axial DTI slice location. Figure $3 \mathrm{~b}$ shows the FA maps with principal diffusion directions from slice 4 to 10 . Multiple compressions existed at $\mathrm{C} 3 / \mathrm{C} 4$ (slice 10), $\mathrm{C} 4 / \mathrm{C} 5$ (slice 8 ) and $\mathrm{C} 5 / \mathrm{C} 6$ (slice 6). The compression at $\mathrm{C} 3 / \mathrm{C} 4$ was the most serious and hyperintense $\mathrm{T} 2$ signal was detected in the cord (Fig 3a). The diffusion directions were also 

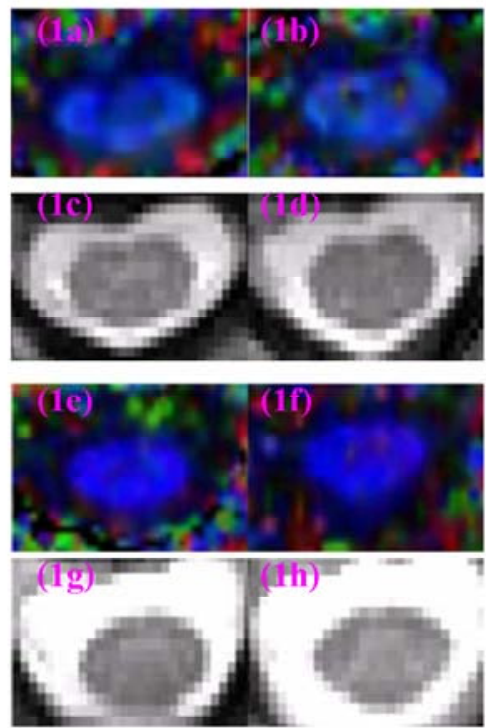

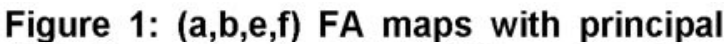
diffusion directions. Different colors represent different diffusion directions (blue - superior/inferior, red - left/right, green - anterior/posterior). (c,d,g,h) the corresponding $\mathrm{T} 2 \mathrm{~W}$ anatomical imaging.

altered by the compression (Fig 3b). Tractography was not possible at the compression sites due to the great alternation of diffusion eigenvectors.

Figures 4abcd show different DT parameters of the cords at different slices. It is observed that at the most serious compression site (slice 10), tt and $\lambda 1$ were elevated drastically (about $45 \%$ and $65 \%$ respectively). $\lambda_{(2+3) / 2}$ was also increased. At slice 8 , where the compression was less serious, increased $\mathrm{tt}, \lambda 1$ and $\lambda_{(2+3) / 2}$ could still be detected but with a smaller extent. On the other hand, FA decreased at less severed compression (slices 6 and 8) but increased in severed compression (slice 10).

On the other hand, $\lambda \mathrm{a}$ and $\lambda \mathrm{r}$ calculated by diffusion eigenvector-based method were compared quantitatively with the conventional method (Fig 5ab). Our data showed that there were remarkable deviations in $\lambda \mathrm{a}$ and $\lambda \mathrm{r}$ at slice 8 between our method and the conventional method. Conventional method overestimated $\lambda \mathrm{a}$ and underestimated $\lambda \mathrm{r}$ at this slice. At slice $10, \lambda \mathrm{a}$ and $\lambda \mathrm{r}$ showed no obvious difference using our method and the conventional method. It means that large diffusion strength was detected in a direction more parallel to the spinal cord at slice 10 .

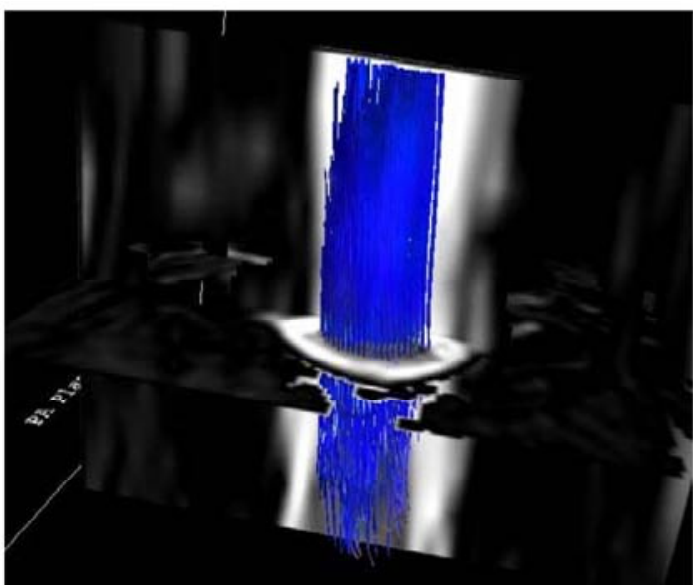

Figure 2: Tractography result showed that fibers ran parallel to the spinal cord.
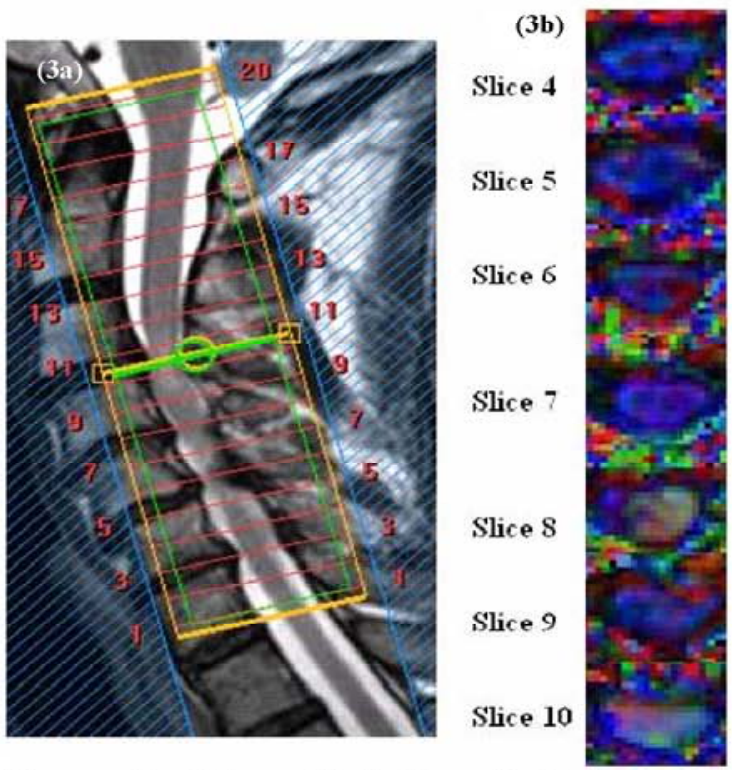

Figure 3: (a) sagittal T2-weighted image with slice location showing multiple compressions and the location of T2 hyperintense signal. (b) FA maps with principal diffusion directions at slice 4-10. Diffusion direction changes at slices 8 and 10. (blue - superior/inferior, red - left/right, green - anterior/posterior)

\section{Discussion}

A protocol for spinal diffusion tensor calculation and tractography has been tested in 3T Achieva system. The combination of small field of view, reduced matrix size and short TE greatly enhanced the image quality. The FA maps (figures la,b,e,f) showed 
consistent low FA region at the anterior part of the spinal cord connecting along all spinal levels. The position of the structure correlated well with the grey matter in the anatomical images (figures lc,d,g,h). DTI showed much better contrast of the grey/white matter than anatomical imaging due to the great difference in diffusion characteristics between grey and white matters. The tractography result (figure 2) showed that the fibers traced ran parallel to the cord corresponding with the spinal neuronal anatomy in normal subjects. The orientation of the fiber bundles traced and the lower anisotropy detected in the grey matter suggested that 3T spinal DTI can show structural information of the cervical spinal cord with high quality. Since axial DTI has high homogeneity in the slice direction, more accurate tensor information along the spinal cord can be obtained.

In CSM, Our results showed that the principal diffusion directions, $\mathrm{tt}, \lambda 1$ and $\lambda_{(2+3) / 2}$ were affected at site of compression in CSM (figure $4 \mathrm{bcd}$ ). Diffusivities are more sensitive to pathophysiological changes in CSM than FA. At the level of serious compression, the diffusivity in a particular direction changed a lot due to destruction of the membranes[4] or abnormal CSF movement[4], leading to a drastic change in diffusion eigenvector and eigenvalues. This explained why tractography was not possible in CSM and why $\mathrm{FA}$ increased at serious compression in our case. On the other hand, FA decreased at less severed compression. It was because the diffusivity change in a particular direction was not as great as in the case of severed compression. Our data showed that FA varied differently according to the compression. Therefore, tt, $\lambda 1$ and $\lambda_{(2+3) / 2}$ are probably more appropriate and sensitive DT parameters for assessing CSM.

In differentiating between $\lambda \mathrm{a}$ and $\lambda \mathrm{r}$, our diffusion eigenvector-based method showed that at the levels of compression, different quantitative results may be obtained compared with the conventional method (figures 5ab). On the other hand, at the levels without compression, our method is almost the same as the conventional one. This proves that in site of compression, $\lambda \mathrm{a}$ can no longer be defined as $\lambda 1$, and $\lambda \mathrm{r}$ as $\lambda_{(2+3) / 2}$. The large diffusivity change in CSM may lead to a drastic increase in the radial diffusion component which may become even larger than the axial component, which will then be mistakenly interpreted as $\lambda \mathrm{a}$ in conventional DT calculation. Therefore, in order to differentiate between $\lambda \mathrm{a}$ and $\lambda \mathrm{r}$ correctly, the diffusion eigenvectors have to be taken into account.
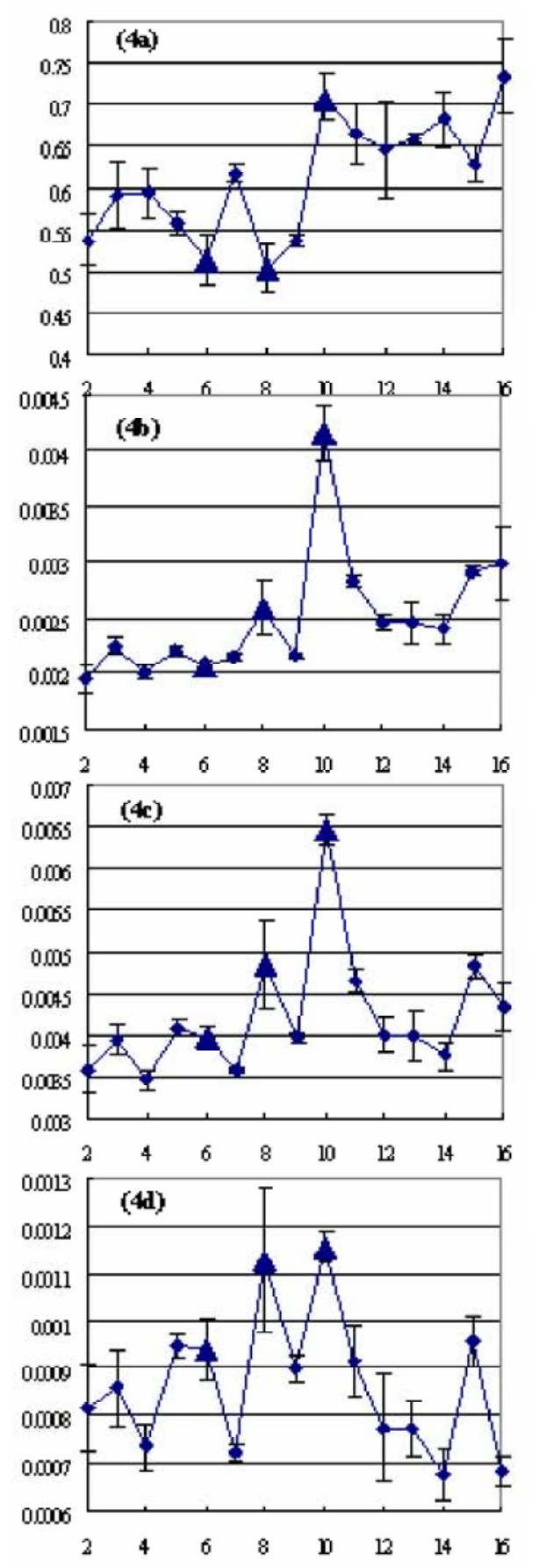

Figure 4: (a) FA (b) tt (c) $\lambda 1$ (d) $\lambda_{(2+3) / 2}$ at different spinal levels. (Y-axis - different DT parameter values with standard error of the mean of the three DT scanning, $X$ axis - slice number, triangle compressed level) 


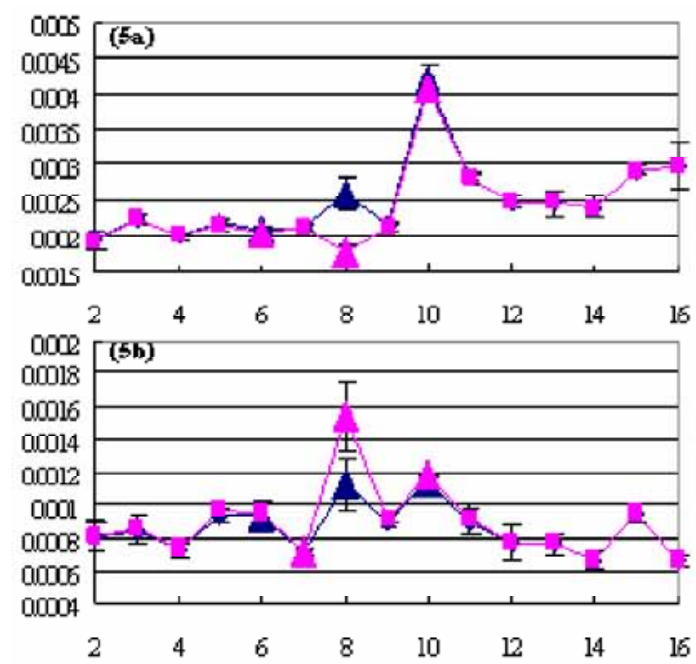

Figure 5: (a) $\lambda \mathrm{a}$ (b) $\lambda \mathrm{r}$ at different spinal levels of the CSM patient. ( $Y$-axis - diffusion strength with standard error of the mean, $X$ axis - slice number, triangle - compressed level, blue line - conventional method, pink line-diffusion eigenvector-based method)

As it has been suggested that the increase in $\lambda \mathrm{r}$ is associated with demyelination rather than axonal damage[8], slice 8 was probably subjected more to demyelination by observing the actual increase in $\lambda \mathrm{r}$ by using our method (figure. $5 \mathrm{~b}$ ). At slice 10, no obvious difference in $\lambda \mathrm{a}$ and $\lambda \mathrm{r}$ using our method and the conventional method may imply that there is abnormal transmedullary movement of the CSF[4] in axial direction.

\section{Conclusions}

A spinal DTI protocol has been developed and tested on healthy subjects and a CSM patient at a 3T system. Clear grey/white contrast was obtained from the spinal FA maps in normal sunjects. Tractography showed fiber running along the spinal cord. All these suggested that our 3T spinal DTI can show structural information of the cervical spinal cord with high quality. In CSM, spinal DTI demonstrated different diffusion characteristics at the sites of compression which showed the potential clinical application of 3T spinal DTI. The proposed diffusion eigenvector-based method was also able to differentiate between $\lambda \mathrm{a}$ and $\lambda \mathrm{r}$ even when there was a drastic diffusivity change at the site of compression in CSM. All these may facilitate better understanding of the pathophysiology of CSM. Studies on more CSM patients will be conducted in the near future to further confirm these findings.

\section{References}

[1] M. Hori, T. Okubo, S. Aoki, H. Kumagai, and T. Araki, "Line scan diffusion tensor MRI at low magnetic field strength: Feasibility study of cervical spondylotic myelopathy in an early clinical stage," J Magn Reson Imaging, 2005.

[2] A. Demir, M. Ries, C. T. Moonen, J. M. Vital, J. Dehais, P. Arne, J. M. Caille, and V. Dousset, "Diffusion-weighted MR imaging with apparent diffusion coefficient and apparent diffusion tensor maps in cervical spondylotic myelopathy," Radiology, vol. 229, pp. 37-43, 2003.

[3] H. Mamata, F. A. Jolesz, and S. E. Maier, "Apparent diffusion coefficient and fractional anisotropy in spinal cord: age and cervical spondylosis-related changes," J Magn Reson Imaging, vol. 22, pp. 38-43, 2005.

[4] M. Ries, R. A. Jones, V. Dousset, and C. T. Moonen, "Diffusion tensor MRI of the spinal cord," Magn Reson Med, vol. 44 , pp. 884-92, 2000.

[5] T. Banasik, M. Hartel, A. Kieltyka, M. Konopka, T. Skorka, and A. Jasinski, "DTI Study of Patients with Cervical Spondylotic Myelopathy," presented at Proc. 13th ISMRM, Miami, U.S.A, 2005.

[6] "FiberTracking in PRIDE," 4 ed: Philips Medical Systems.

[7] H. Jiang and S. Mori, "DTIStudio," 2.02 ed: Johns Hopkins University, 2004.

[8] S. K. Song, J. Yoshino, T. Q. Le, S. J. Lin, S. W. Sun, A. H. Cross, and R. C. Armstrong, "Demyelination increases radial diffusivity in corpus callosum of mouse brain," Neuroimage, vol. 26, pp. 132-40, 2000 